\section{Gas phase detection of Chemical Warfare Agents CWAs with portable Raman}

\author{
3 Marta Lafuente ${ }^{\mathrm{a}, \mathrm{b}}$, Diego Sanz ${ }^{\mathrm{a}, \mathrm{b}}$, Miguel Urbiztondo ${ }^{\mathrm{c}}$, Jesús Santamaría

\section{Abstract}

The development of SERS substrates for chemical detection of specific analytes requires appropriate selection of plasmonic metal and the surface where it is deposited. Here we deposited $\mathrm{Ag}$ nanoplates on three substrates: i) conventional $\mathrm{SiO}_{2} / \mathrm{Si}$ wafer, ii) stainless steel mesh and iii) graphite foils. The SERS enhancement of the signal was studied for Rhodamine 6G (R6G) as common liquid phase probe molecule. We conducted a comprehensive study with $\lambda=532,633$ and $785 \mathrm{~nm}$ on all the substrates. The best substrate was investigated, at the optimum laser $785 \mathrm{~nm}$, for gas phase detection of dimethyl methyl phosphonate (DMMP), simulant of the G-series nerve agents, at a concentration of $2.5 \mathrm{ppmV}\left(14 \mathrm{mg} / \mathrm{m}^{3}\right)$. The spectral fingerprint was clearly observed; with variations on the relative intensities of SERS Raman bands compared to bulk DMMP in liquid phase reflects the DMMP-Ag interactions. These interactions were simulated by Density Functional Theory (DFT) calculations and the simulated spectra matched with the experimental one. Finally, we were detected the characteristics DMMP fingerprint with hand-held portable equipment. These results open the way for the application of SERS technique on real scenarios where robust, light-weight, miniaturized and simple to use and cost-effective tools are required by first responders.

Keywords: Gas phase detection, SERS, portable Raman, CWAs, trace level 


\section{Introduction}

The nerve gas attacks with sarin in Matsumoto (1994) and Tokyo (1995) and in the armed conflict in Syria (Goutha 2013 and Khan Shaykhun 2017) are recent and representative examples of the present world threat spectrum. Chemical Warfare Agents (CWAs), initially developed in the First World War, include vesicant and blister agents such as phosgene (PD) and mustard gas (HD) and nerve agents, as sarin (GB) and venomous agent $\mathrm{X}(\mathrm{VX})$. The nerve agents are highly toxic due to the irreversible binding with the nerve sites responsible for acetylcholinesterase breakdown, a necessary process in neurotransmission. Sarin gas presents the highest toxicity with a IDLH (Immediately Dangerous to Life or Health) of $0.1 \mathrm{mg} \cdot \mathrm{m}^{-3}$. These CWAs are able to spread out in the atmosphere, creating a toxic scenario in few seconds. The early detection and identification of these agents in gas phase is essential for the safety of first responders and for the efficient evacuation of threatened public spaces.

Surface Enhanced Raman Spectroscopy (SERS) is a technique capable of label-free ultrasensitive vibrational "fingerprinting", and as such it is recognized as highly interesting method for explosive and chemical threat detection [1]. The technique has also become suitable for on-site detection thanks to the development of portable equipment with adequate spectral resolution (ranging from 7 to $12 \mathrm{~cm}^{-1}$ ) and low weight (330 to $2450 \mathrm{~g}$ ) at reasonable prices, ca. 10 thousand euros without spectral library. However there are still several challenges to be overcome before this technique can be implemented as "off-the-shelf" solution for CWA detection.

SERS mainly relies on the enhancement of the Raman signal due to the plasmon resonance of a metallic nanostructure. This effect only occurs when the target molecule is in close contact to the metal, and for this reason an interaction i.e. physical or chemical adsorption is required between the molecule and the metal. The magnitude of the enhancement depends on several parameters including composition, size, morphology, topology, surface distribution, and dielectric environment of the metallic nanostructure on substrate surface. Many efforts have been devoted in SERS field for the development of optimum SERS substrates ranging from fancy shapes of nanoparticles with sharp edges to ordered nanostructures whose the shape and spacing was optimized with simulation and then on fabricated in cleanroom. Other key factors 
for the highly sensitive detection of molecules include the cross-section of the molecule, its stability, interactions with the SERS substrate and its solid, liquid or gaseous state.

Most of the SERS applications in the open literature refer to the detection in liquid phase and only few reports for gas phase detection at concentrations below $10 \mathrm{ppmV}$ could be found. Van Duyne and coworkers reported the detection of $8 \mathrm{ppmV}$ of benzene thiol on a SERS substrate consisting of well-ordered silica nanospheres coated with a $200 \mathrm{~nm}$ thick Ag film [2]. A recyclable surface-enhanced Raman scattering (SERS) substrate, consisting of $\mathrm{Ag}$ nanorods coated with an ultrathin $\mathrm{HfO}_{2}$ shell (Ag

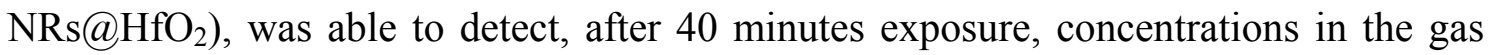
phase as low as 20ppb of the molecule 2-Naphthalenethiol (2-NAT). Then substrate was easily regenerated by heating at $250^{\circ} \mathrm{C}$ for 25 seconds [3]. The SERS detection of volatile organic compounds (VOCs) biomarkers in human breath, such as acetone in the case of diabetes or hydrogen cyanide in patients with cystic fibrosis, is gaining of importance as non-invasive tool in primary screening diagnosis. The group of Boisen detected 5ppmV of hydrogen cyanide in gas phase [4] and later on P. aeruginosa cultures [5]. The term of plasmonic nose was recently coined for a SERS substrate that consists on a core-shell structure of Ag nanocubes encapsulated in porous metal organic framework MOF, in particular ZIF-8 [6]. The VOCs adsorbed on the porous layer formed a 3D confinement space for the molecules near the plasmonic structure. In this system $200 \mathrm{ppmV}$ of toluene were detected and the detection limit could be lowered to $50 \mathrm{ppb}$ for 2-nitrotoluene, a molecule with higher cross section. Another recent example of low concentration in gas phase was the detection of $10 \mathrm{ppmV}$ of benzene. In this case the strategy for decreasing the detection limit involved deep cooling of the SERS substrate down to $-80^{\circ} \mathrm{C}$, to promote adsorption and condensation of the molecules [7]. Recently, a 3D porous substrate successfully detected for the first time $0.1 \mathrm{ppmV}$ of $\mathrm{NO}_{2}$ in the gas phase with a hand-held Raman detector [8]. The high surface area employed consisted of a 3D multilayer structure made of $\mathrm{Au}$ coated-Ag nanowires (AgNWs) forming lots of random hot spots in the cross points of the fibers. In our laboratory, the detection of $625 \mathrm{ppbV}$ of DMMP, a surrogate molecule of the G-series nerve agents which are of particular concern due to its extreme toxicity and persistence, was recently reported using a substrate consisting of self-assembled AuNPs coated with a citrate layer that acted as an effective trap for the target molecules [9]. The above results illustrate the importance of substrate selection for gas phase using SERS. It must 
provide high electromagnetic enhancement (EF), but also chemical affinity towards the target molecule while avoiding band interference with the functional groups responsible for this affinity.

In this work we selected $\mathrm{Ag}$ nanoplates as the plasmonic material to be used in DMMP detection in view of four reasons: i) the optical properties of $\mathrm{Ag}$ (real and imaginary parts of the dielectric function), that makes this metal the main candidate to get interesting optical effects, including plasmon resonances [10]; ii) the specific interaction of DMMP with Ag through the P-O bond, as already reported in the first study of DMMP detection in roughened Ag electrodes [11]; iii) the electromagnetic field enhancement at the tips and edges of the nanoplates; and finally, iv) the coincidence of the plasmon resonance band of Ag nanoplates with the excitation laser wavelength used for the Raman measurements, $785 \mathrm{~nm}$. We have compared the response of the synthesized $\mathrm{Ag}$ nanoplates on three different substrates, $\mathrm{SiO}_{2} / \mathrm{Si}$, stainless steel mesh and graphite foil. The SERS response was analyzed using Rhodamine (R6G) as probe molecule with lasers of $\lambda=532,633$ and $785 \mathrm{~nm}$. The same substrates were also studied for the detection of $2.5 \mathrm{ppmV}$ of DMMP in gas phase with a benchtop and portable Raman equipments. The interactions of the DMMP molecule with the Ag surface were simulated using Density Functional Theory (DFT) calculations carried out by Gaussian09 quantum chemistry program [12] to explain the relative intensities variation of the characteristic Raman vibrational modes.

\section{Materials and methods}

\subsection{Materials}

Silver nitrate $\left(\mathrm{AgNO}_{3}, 99.9999 \%\right)$, sodium citrate tribasic dehydrate $(>99 \%)$, hydrogen peroxide solution $\left(\mathrm{H}_{2} \mathrm{O}_{2}, 30 \% \mathrm{wt}\right)$, potassium bromide $(\mathrm{KBr},>99 \%)$, sodium borohydride $\left(\mathrm{NaBH}_{4},>99 \%\right)$, poly(diallyl dimethylammonium (PDDA, 20\% wt), rhodamine 6G (99\%) and dimethyl methylphosphonate (DMMP, 97\%) were purchased from Sigma-Aldrich. All solutions were prepared in distilled water. Acetone and isopropyl alcohol were also purchased from Sigma-Aldrich. DMMP vapours were generated using a calibrated permeation tube (MT-PD-Experimental 107-100-7845HE3-C50, $126.78 \mathrm{ng} / \mathrm{min} \pm 4.81 \mathrm{ng} / \mathrm{min}$ at $80^{\circ} \mathrm{C}$ ) from VALCO.

\subsection{Preparation of SERS substrates}


The Ag nanoplates (Ag nPlates) were synthesized according to a previously reported method [13]. Briefly, in a $20 \mathrm{~mL}$ vial, the following aqueous solutions were sequentially added while stirring: $\mathrm{H}_{2} \mathrm{O}(8.5 \mathrm{~mL}) ; \mathrm{AgNO}_{3}(3.75 \mathrm{~mL}, 0.5 \mathrm{mM})$; sodium citrate $(1 \mathrm{~mL}$, $25 \mathrm{mM}) ; \mathrm{H}_{2} \mathrm{O}_{2}(28.2 \mu \mathrm{L})$; $\mathrm{KBr}(10 \mu \mathrm{L}, 1 \mathrm{mM})$. Finally $\mathrm{NaBH}_{4}(1.25 \mathrm{~mL}, 10 \mathrm{mM})$ was quickly added to the solution to cause Ag reduction. A colour transition from yellow to blue could be observed in the solution after approximately two minutes, indicating plate formation. Ag nPlates solutions were then centrifuged afterwards for 20 minutes at $21,000 \mathrm{rpm}$, and the supernatant was centrifuged again. The obtained precipitates were resuspended in water and citrate was added to obtain a solution with a final concentration of $80 \mathrm{mg} / \mathrm{L}$ of $\mathrm{Ag}$ and $2.5 \mathrm{mM}$ of sodium citrate.

The Ag nPlates were deployed on three different supports (4mm x 8mm): $\mathrm{SiO}_{2} / \mathrm{Si}$ chip (Sil'Tronix $1 \mu \mathrm{m}$ of wet thermal $\mathrm{SiO}_{2}$ wafer), stainless steel mesh (see SEM image in Fig. S1) and graphite foil (RivaTherm-HD type from Kempchen). Figure S2 shows digital photographs of the three different supports. Before the deposition the supports were sequentially washed with acetone, isopropanol and water during $10 \mathrm{~min}$ each, in an ultrasound bath. After, SERS substrates were immersed in an aqueous solution of PDDA $(0.2 \% \mathrm{v} / \mathrm{v})$ during 4 hours, washed with distilled water and dried for 10 minutes at $100{ }^{\circ} \mathrm{C}$. Finally, the supports were immersed in the Ag nplates suspension overnight and allowed to dry at room temperature, to obtain the SERS substrates. In the case of graphite, before immersing in the PDDA solution the surface was peeled-off with scotch tape. The obtained SERS substrates are denoted as nPlates@ $\mathrm{SiO}_{2} / \mathrm{Si}$, Ag nPlates@SS and Ag nPlates@graphite for the remainder of this work.

\subsection{Material Characterization}

Size distribution and morphology of Ag nPlates were studied by TEM (FEI Tecnai T200). Scanning electron microscopy (SEM) images were obtained using a FEG INSPECT 50. Five SEM images of each SERS substrates after coating were analysed to assess the Ag nPlates density on the surface using ImageJ analysis software. A UV-Vis Spectrophotometer Varian Cary 50 Spectrometer was used to measure the absorbance properties of Ag nPlates in solution and Surface Plasmon Resonance (SPR) spectra of the Ag nPlates coated on a glass substrate following the same protocol described above for the other substrates. The measurements of the UV-Vis absorption spectra for the Ag nPlates coated on a stainless steel mesh and graphite substrate were performed in a 
Jasco V-670 spectrometer equipped with a diffuse reflectance chamber. The measurements of the solid surfaces were allways obtained with white $\mathrm{BaSO}_{4}$ as reference for the background.

\subsection{Raman Spectroscopy for characterization of SERS substrates.}

An Alpha 300 Raman spectrometer of WITec was used with a confocal optical microscope (480nm as lateral spatial resolution, $2 \mathrm{~cm}^{-1}$ spectral resolution). RamanSERS spectra were collected in backscattering geometry. Excitation of the samples was carried out with lasers $532 \mathrm{~nm}, 633 \mathrm{~nm}$ and $785 \mathrm{~nm}$ at room temperature and applying a irradiance of $0.13 \mathrm{~mW} / \mu \mathrm{m}^{2}, 0.67 \mathrm{~mW} / \mu \mathrm{m}^{2}$ and $0.88 \mathrm{~mW} / \mu \mathrm{m}^{2}$, respectively.

The analytical enhancement factor (AEF) [14], of the different substrates, was calculated according to equation (1). R6G was selected as probe molecule monitoring its C-C stretching mode displaced at $1512 \mathrm{~cm}^{-1}$.

$$
A E F=\frac{\frac{I_{S E R S}}{C_{S E R S}}}{\frac{I_{\text {Raman }}}{C_{\text {Raman }}}}
$$

Where $\mathrm{C}_{\text {Raman }}$ and $\mathrm{C}_{\mathrm{SERS}}$ are the $\mathrm{R} 6 \mathrm{G}$ concentration in the Raman measurements and SERS conditions, respectively. And $I_{\text {Raman }}$ and $I_{S E R S}$ are the normalized intensity values (cts $\cdot \mathrm{mW}^{-1} \cdot \mathrm{s}^{-1}$ ) of the $1512 \mathrm{~cm}^{-1}$ band for normal Raman and SERS measurements, respectively.

A droplet of $2 \mu \mathrm{L}$ aqueous solution R6G $10 \mu \mathrm{M}$ was deposited on the SERS substrate and dried under ambient conditions. The R6G spectrum of the SERS substrate was measured in ten different points of the dried droplet and the intensity of the peak at $1512 \mathrm{~cm}^{-1}$ was averaged. The normal Raman R6G spectrum was measured focusing the laser beam, $633 \mathrm{~nm}$ and $785 \mathrm{~nm}$, in the aqueous solution R6G $1 \mathrm{mM}$. For the $532 \mathrm{~nm}$ laser line, the R6G droplet was dried before acquisition to avoid fluorescence effects.

\subsection{Experimental set-up for gas phase measurements}

SERS experiments for detecting DMMP in gas phase were conducted in a gas cell $\left(2.7 \times 10^{-2} \mathrm{~cm}^{3}\right)$, where a gas stream, $10 \mathrm{ml} \mathrm{STP} / \mathrm{min}$, containing $2.5 \mathrm{ppmV}\left(14 \mathrm{mg} / \mathrm{m}^{3}\right)$ of DMMP in nitrogen was fed continuously. DMMP vapours were generated using a calibrated permeation tube as described in our previous work [9]. Two Raman equipments were used: the benchtop equipment described above (Alpha 300 Raman spectrometer, WITec) and a portable Raman BWTEK i-Raman pro system $\left(6 \mathrm{~cm}^{-1}\right.$ 
spectral resolution). In the latter case, an excitation wavelength of $785 \mathrm{~nm}$ with a power laser of $280 \mathrm{~mW}\left(0.035 \mathrm{~mW} / \mu^{2}\right)$, 1s of integration time and averaging 100 spectra were used for all the measurements. A schematic drawing together with a picture of the set-up with the portable Raman and the gas cell for measurements are presented in Fig. 1.

a)

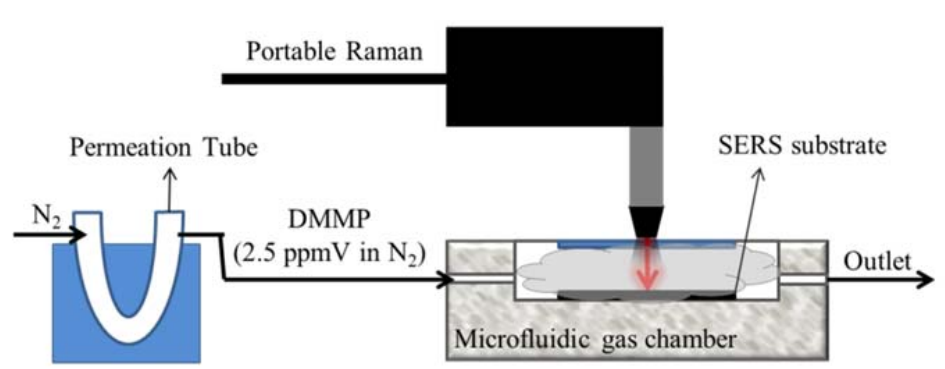

b)

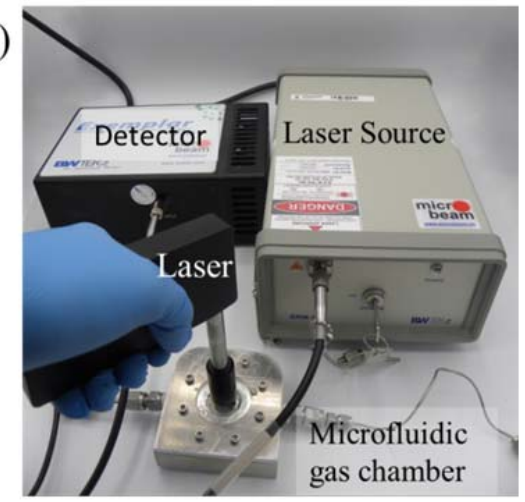

Fig. 1. a) Scheme of the experimental set-up used for detection of CWA vapours. b) Digital photograph of the real set-up including the portable Raman and the microfluidic gas chamber.

\subsection{DFT calculations}

Geometry optimizations and frequency analysis calculations for the organophosphorous compound and Ag atoms were undertaken using DFT methods. All the calculations were carried out using the Gaussian 09 [12] software package.

The ground state geometries were optimized employing the density functional theory (DFT) $[15,16]$ with the B3LYP hybrid functional (Becke's gradient-corrected exchange correlation in conjunction with the Lee-Yang-Parr correlation functional with three parameters) and the $6-311++\mathrm{G}(\mathrm{d}, \mathrm{p})$ one-electron atomic basis sets. The adsorbed DMMP molecules and the Ag atoms were calculated with the $6-311++\mathrm{G}(\mathrm{d}, \mathrm{p})$ basis set and the LANL2DZ basis set considering a pseudo potential, respectively.

\section{Results and Discussion}

\subsection{Characterization of SERS substrates}

The synthesis of the Ag nPlates was reproducible and in all the cases the UV-Vis spectrum (Fig. 2a) displayed the main broad band at around 700nm, assigned to the inplane dipole plasmon resonance. The other two LSPR bands at $460 \mathrm{~nm}$ and $331 \mathrm{~nm}$ are 
assigned to the in-plane quadrupole and out-of-plane quadrupole plasmon resonances of 214 triangular nanoplates, respectively [17]. We observed that during storage at room temperature and in the darkness, the peak intensity progressively decreased and blue shifted to $640 \mathrm{~nm}$ after 30 days (see Fig. 2 a). The Localize Surface Plasmon Resonance (LSPR) is very dependent on the shape and size of the nanoparticles, and this evolution is probably caused by surface atomic migration within the Ag nPlate that evolves towards more thermodynamically stable forms [18]. In our case this involves a progressive smoothening of the plates, since the round shape (111), is more stable than tips (110) in triangular shape. The analysis of TEM images for freshly made samples (Fig. 2b) shows a mixture of $\mathrm{Ag}$ nanoparticles and nanoplates resulting in an average yield of $85 \%$ to plates with an edge of $38 \pm 15 \mathrm{~nm}(\mathrm{~N}>500)$.
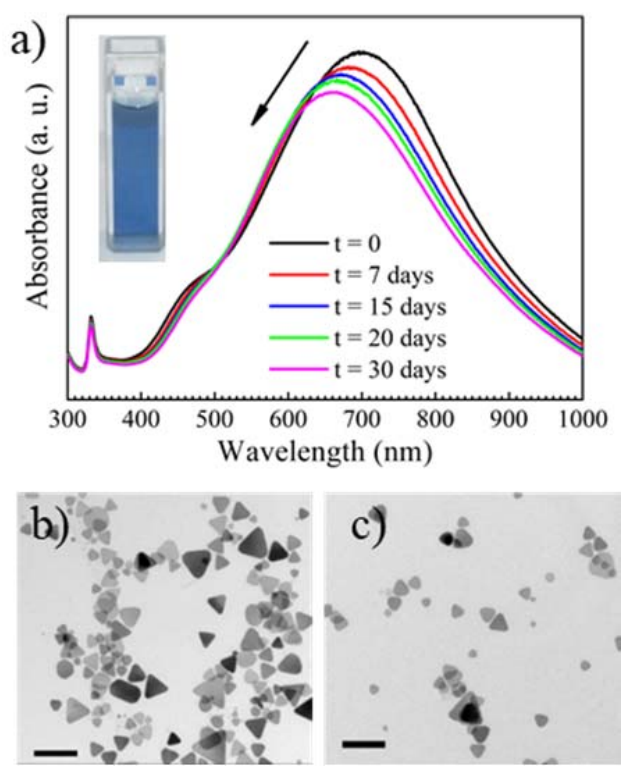

Fig 2. a) UV Vis spectra of aqueous solution containing Ag nPlates: evolution with time during storage. Insert: digital photograph of Ag nanoplates solution. TEM image of synthesized Ag plates: b) freshly made and c) aged 30 days. Scale bar $100 \mathrm{~nm}$.

The SEM images presented in Fig. 3 show a homogeneous distribution of the Ag nPlates deposited on different surfaces. The analysis of the SEM images resulted in a similar surface coverage for the three substrates $42 \pm 5 \%\left(\mathrm{SiO}_{2} / \mathrm{Si}\right.$ chip $), 41 \pm 5 \%$ (stainless steel mesh) and $44 \pm 3 \%$ (graphite foil) These values are higher than the $30 \%$ coverage that we previously found with $\mathrm{Au}$ NPs deposited on $\mathrm{SiO}_{2} / \mathrm{Si}$ surfaces [9]. This could be attributed to the planar structure of the Ag nanoplates, versus the spherical $\mathrm{Au}$ nanoparticles. It is also possible to observe densely packed aggregates of nanoplates, 
resulting in close contact of their edges that will result in couplings between LSPRs of the metallic nanoplates [19]. The UV-Vis spectrum for the Ag nPlates deposited on the glass substrate broadens and the maximum is blue shifted compared to the nanoplate solution (see Fig. S3). This observation could be attributed to the differences in the dielectric permittivity either of the propagating media of the light, changing from water in the liquid solution to air in the case of the coated substrates [20] or differences in the differences in the properties of underlying substrates [21,22]. The broadening of the spectrum favors the coupling of the plasmon with the different lasers and specially with the $785 \mathrm{~nm}$ due to the blue shifting observed. In the case of the non-transparent substrates stainless steel and graphite, the diffuse reflectance UV-Vis spectra (Figure S3) showed an increase in the absorption band of the coated substrates in the region above $700 \mathrm{~nm}$ corresponding to the SPR of the silver plates.
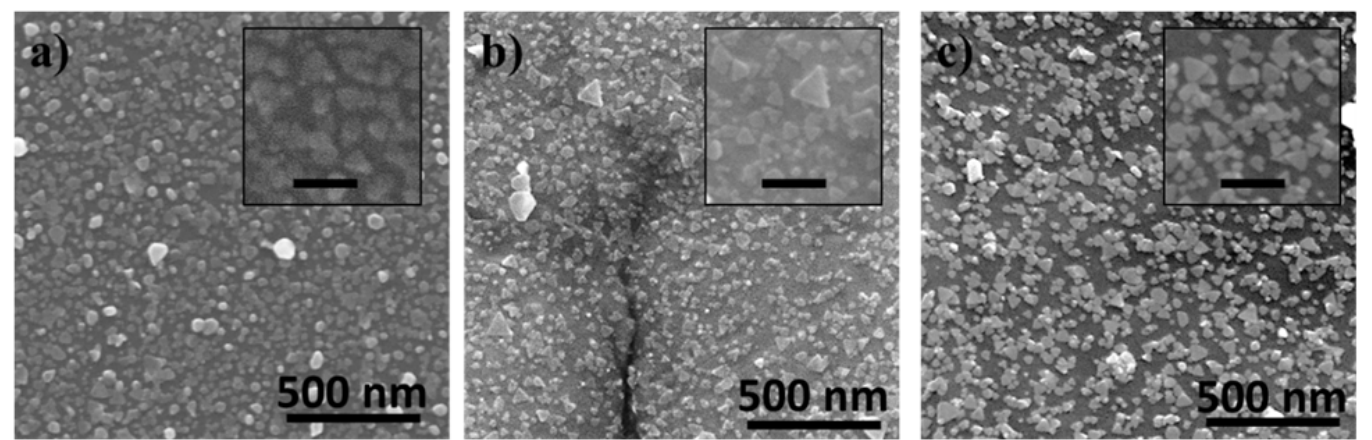

Fig. 3. SEM images of Ag nanoplates deposited on these SERS substrates: a) $\mathrm{SiO}_{2} / \mathrm{Si}$; b) stainless steel mesh; c) graphite foil. Insert scale $50 \mathrm{~nm}$.

The three substrates were first characterized with R6G as a probe molecule with three different lasers 532, 633 and $785 \mathrm{~nm}$. The as collected spectra, without any modifications (i.e. baseline corrections and smoothing) are presented in Fig. 4. It is immediately apparent that the highest intensity and lower signal to noise ratio for R6G it is observed for the $532 \mathrm{~nm}$ laser. The Raman intensity depends on the molecules under study and, when the analyte is a chromophore, close in energy to the frequency of the excitation, resonant Raman scattering (RRS) occurs. The RRS intensities can be $10^{6}$ larger than normal (off-resonance) Raman intensities [10], in this case we should called the technique SERRS instead of just SERS. When the molecule is adsorbed on the surface of the metal, the strong fluorescence of R6G, is quenched to a certain extent due to non-radiative interactions with the metal surface. When comparing the raw data of the spectra at $532 \mathrm{~nm}$, for the three different substrates (Fig. 4a), the fluorescence of 
262 R6G could observed as a broad band in the spectrum, centered at around $600 \mathrm{~cm}^{-1}$. The

263 quenching of this signal is clearly more effective for the case of the graphite substrate. It

264 has been reported that not only graphene, but also other carbonaceous materials could

265 be considered as good SER(R)S substrates due to resonance effect and quenching 266 fluorescence either via charge transfer or energy transfer [23]. In fact, for the $\mathrm{SiO}_{2} / \mathrm{Si}$ 267 substrate, the one with the lowest conductivity $\left(10^{-1} \mathrm{~S} / \mathrm{m}\right)$ we observed the lowest EF 268 and SERS gain (Fig. 4) for all the wavelengths tested.

269 In the case of the excitation with the $633 \mathrm{~nm}$ laser, where the energy of the laser is 270 still near to the excitation of the chromophore, we can still observe the R6G spectra 271 although with lower intensities for the three supports (Fig. 4b). In the case of $785 \mathrm{~nm}$ 272 laser line, this wavelength matches with the plasmon resonance of the Ag nPlates (see 273 Fig. 2 and Fig. S3), and the spectrum of the R6G molecule could only clearly 274 distinguished for the graphite support (Fig. 4c), most probably due to charge transfer 275 from the electrons of the plasmonic metal to the graphite. The easily available, low cost 276 graphite material could an alternative support for the deposition of active SERS 277 nanostructures being capable of making an additional contribution to the enhancement 278 of the signal by different mechanisms, including fluorescence quenching, charge and 279 energy transfer and also probably resonant effects of this support. This is in accordance 280 with the observations presented by Sil et al. [23] for different carbonaceous materials 281 (charcoal, graphite, MWCNT and GO).

282 From averaging 10 different spots in each experimental condition (excitation 283 wavelength and substrate), the analytical enhancement factor (AEF) was calculated 284 (Fig. 4d). In the three studied substrates at the excitation wavelength of $785 \mathrm{~nm}$ reports the highest analytical enhancement factor was obtained, even for the spectra on Ag 286 nPlates@SiO $\mathrm{Si}_{2} / \mathrm{Si}$ and Ag nPlates@SS that is less defined. This result is due to the low 287 signal of R6G in Raman conditions ( $\mathrm{I}_{\text {Raman }}$ ) under excitation wavelength of $785 \mathrm{~nm}$. Fig. 288 S4 shows the Raman spectrum of R6G $1 \mathrm{mM}$ under the three excitations wavelength. 

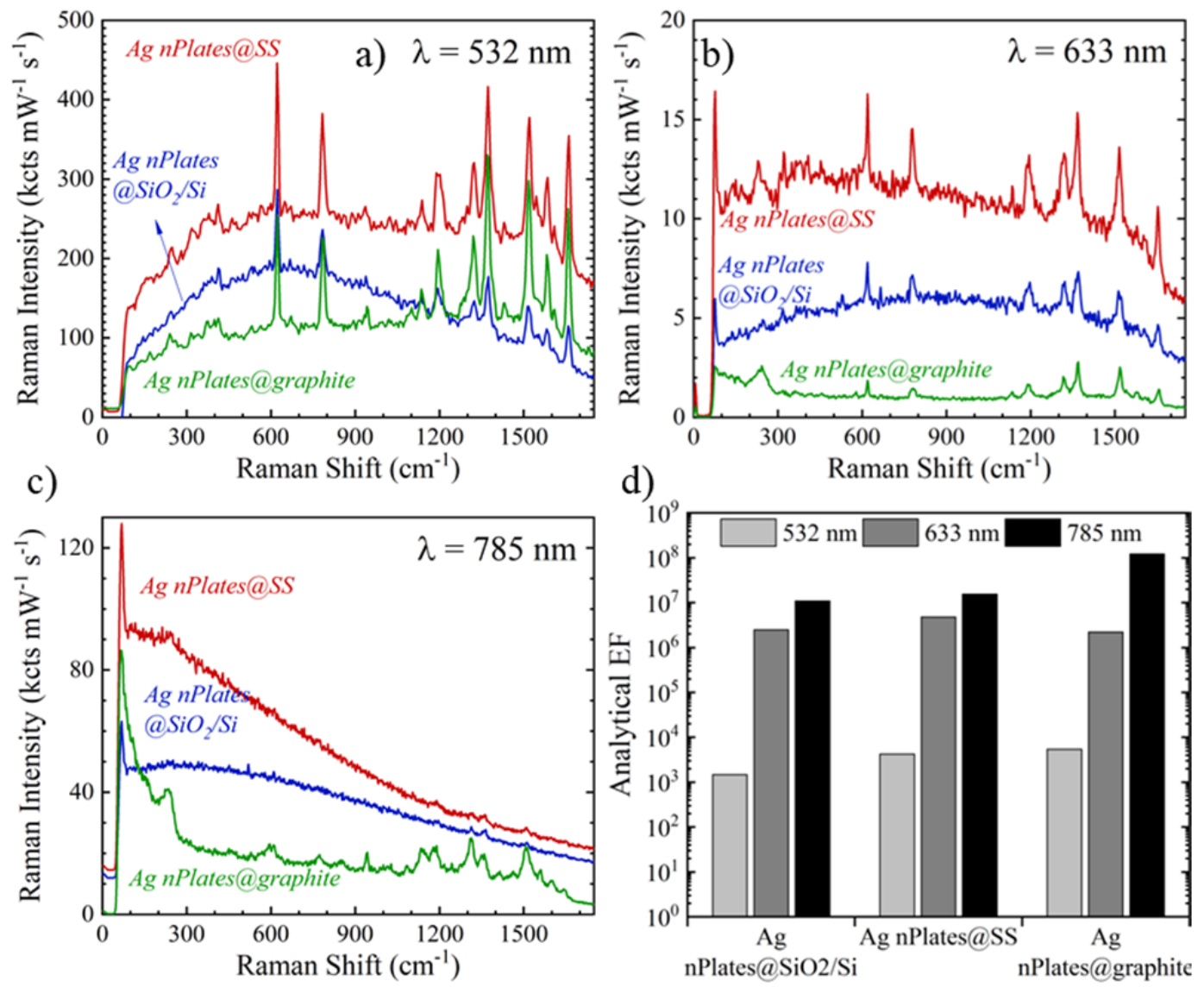

291

292

293

294

295

296

297

298

299

300

301

302

303

304

305

Fig. 4. Raman spectra of R6G $10^{-5} \mathrm{M}$ droplet dried on three different SERS substrates: Ag nPlates@SiO $/$ Si,AgnnPlates@SS andAg nPlates@graphite for different excitation wavelengths: a) $532 \mathrm{~nm}$ : b) $633 \mathrm{~nm}$ and c) 785nm. d) Analytical EF (AEF) calculated for the three SERS substrates and for different excitation wavelengths (532, 633 and $785 \mathrm{~nm}$ ). For better visualization in a) and c) spectra have been vertically translated.

\subsection{CWAs gas phase detection capabilities of the SERS substrates}

The SERS substrates were also tested for the measurement of the simulant nerve agent DMMP in gas phase in the benchtop equipment using the $785 \mathrm{~nm}$ laser and graphite substrate, which resulted in the highest AEF. Furthermore in the case of using $633 \mathrm{~nm}$ and $785 \mathrm{~nm}$ with $\mathrm{SiO}_{2} / \mathrm{Si}$ and stainless steel mesh substrates, it was not possible to get a spectrum. As soon as the laser was focused on the sample, the DMMP molecules were decomposed and a broad band in the region 1450 to $1700 \mathrm{~cm}^{-1}$, corresponding to amorphous carbon was observed. This phenomenon is attributed to the photo-thermal decomposition of the DMMP molecules adsorbed on the enhancing metal surface [24]. 
The Raman spectrum for neat liquid DMMP and the SERS spectra on Ag nPlates@graphite for a concentration of 2.5ppmV DMMP in the gas phase are presented in Fig. 5a and Fig 5b for benchtop and portable Raman equipments, respectively. First of all, it is important to note that the fingerprint of the DMMP molecule is observed with both detectors. However the relative intensity of the characteristic bands differs from the acquired at normal Raman conditions. The Raman spectra recorded by the portable equipment on randomly selected spots are presented in Fig. $5 \mathrm{~b}$. Due to the lower resolution of the portable equipment, $6 \mathrm{~cm}^{-1}$, compared to 2 $\mathrm{cm}^{-1}$ in the benchtop equipment, the bands are broader and could be also slightly shifted. This is clearly observed for the main band of the DMMP liquid measured in both equipments. It is important to note that the spectra were reproducible in all the randomly selected spots, showing three distinctive bands, with similar intensities and assigned to $\mathrm{PO}_{3}, \mathrm{P}-\mathrm{C}$ stretching and $\mathrm{PO}_{2}$ bending (for more details see Table 1). This allowed a clear identification of the DMMP using the hand held instruments, for a gas phase concentration of $2.5 \mathrm{ppmV}$.

Fig. 6a shows the molecular structures of the two investigated DMMP-Ag complexes. The most stable Ag-DMMP geometry, among studied, corresponds with DMMP molecule adsorbed on $6 \mathrm{Ag}$ atoms (in blue) though $\mathrm{P}=\mathrm{O}$ group. A less-stable Ag-DMMP* complex resulting from DMMP approximation of the phosphate group that looks like an umbrella, $\left(\mathrm{PO}_{3}\right)_{\text {umbrella }}$ is also plotted.

Fig. $6 \mathrm{~b}$ compiles the simulated Raman spectra of DMMP molecule and DMMP-Ag (see Fig. 5 and Table 1), simulated Raman spectra show similar bands, although changes in both relative intensities and the position of the bands are clearly noticed. Bands at 504 and $715 \mathrm{~cm}^{-1}$ assigned to $\left(\mathrm{PO}_{3}\right)_{\text {umbrella }}$ bending and to $\left(\mathrm{P}-\mathrm{CH}_{3}\right)$ stretching + $(\mathrm{P}-\mathrm{O})$ stretching $+\left(\mathrm{P}-\mathrm{O}-\mathrm{CH}_{3}\right)$ bending, respectively, are slightly displaced to around 483 and $672 \mathrm{~cm}^{-1}$ (for more details see Table 1). The ratio between these two simulated bands (Intensity at $672 \mathrm{~cm}^{-1} /$ Intensity at $483 \mathrm{~cm}^{-1}$ ) for DMMP molecule and AgDMMP $(\mathrm{P}=\mathrm{O})$ complex is the same. However, the simulated spectrum for Ag-DMMP* $\left(\mathrm{PO}_{3}\right)$ shows an increment in this ratio, similar to the one observed experimentally. This could provide some insight on the adsorption dynamics of DMMP molecules on Ag plates under experimental conditions. Although less energetically favored, this geometry is contributing to the dynamic SERS response. However, it should be noticed 
339 that for simplicity the simulation considers one DMMP molecule and 6 silver atoms 340 while experimentally, a molecule of DMMP may interact not only with several silver 341 atoms but also with other DMMP molecules, increasing the possibilities of $\left(\mathrm{PO}_{3}\right)_{\text {umbrella }}$ $342-$ Ag interactions.
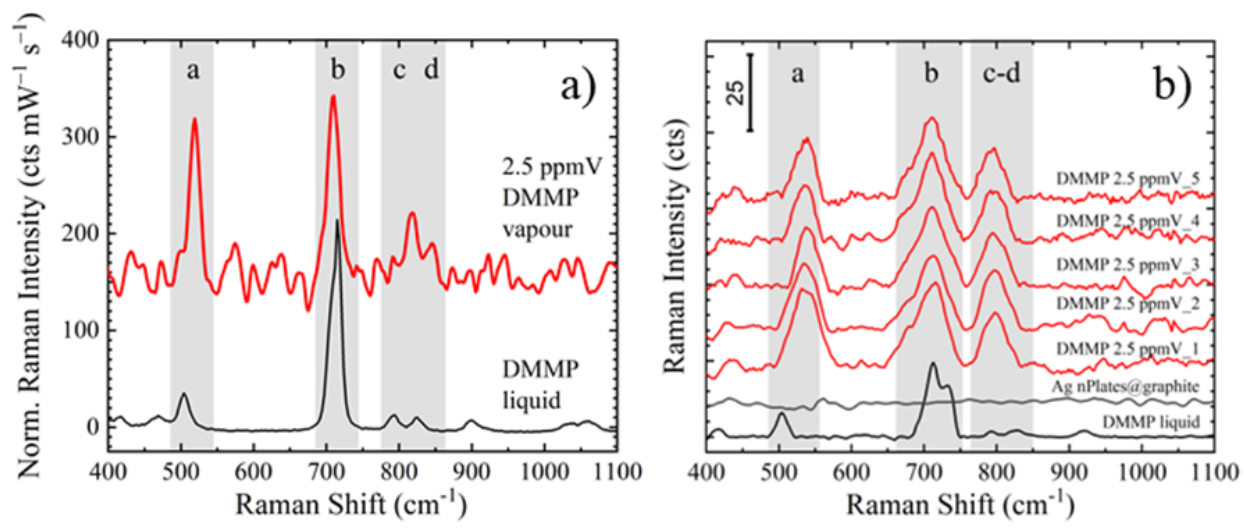

344 Fig 5. Raman spectra of DMMP liquid and DMMP in vapour phase (2.5 ppmV, $\left.14 \mathrm{mg} / \mathrm{m}^{3}\right)$ measured on Ag nplates@graphite using: a) the benchtop equipment and b) the portable equipment. For the portable equipment five different spectra for vapours detection are shown, measured at different random spots on the support, together with the spectrum of $\mathrm{Ag}$ nPlates@graphite. (a-b) Excitation wavelength 785 nm; grey shadows indicate bands assigned to DMMP (more information in Table 1).

Table 1. Temptative assignments of the experimental Raman and SERS bands for DMMP pure liquid and vapor on Ag nPlates@graphite, together with calculated values by DFT simulations.

\begin{tabular}{|c|c|c|c|c|c|c|}
\hline \multirow{3}{*}{ Band } & \multicolumn{5}{|c|}{ Raman Shift of DMMP vibrational modes $\left(\mathrm{cm}^{-1}\right)$} & \multirow{3}{*}{$\begin{array}{c}\text { Temptative } \\
\text { assignment } \\
{[25]}\end{array}$} \\
\hline & \multirow{2}{*}{$\begin{array}{c}\text { Pure liquid } \\
\text { Experimental }\end{array}$} & \multirow{2}{*}{$\begin{array}{l}\text { Calculated } \\
\text { DMMP in } \\
\text { vacuum }\end{array}$} & \multicolumn{2}{|c|}{$\begin{array}{c}\text { DMMP Vapors on } \\
\text { Ag nPlates@graphite: }\end{array}$} & \multirow{2}{*}{$\begin{array}{l}\text { Calculated } \\
\text { Ag- DMMP } \\
\left(\mathrm{PO}_{3}\right)\end{array}$} & \\
\hline & & & $\begin{array}{l}\text { Benchtop } \\
\text { equipment }\end{array}$ & $\begin{array}{c}\text { Portable } \\
\text { equipment }\end{array}$ & & \\
\hline $\mathrm{a}$ & 504 & 483 & 515 & 539 & 469 & Bending $\mathrm{PO}_{3}$ \\
\hline $\mathrm{b}$ & 715 & 672 & 710 & 712 & 663 & Stretching PC \\
\hline $\mathrm{c}$ & 794 & 756 & 814 & 797 & 756 (weak) & Bending $\mathrm{PO}_{2}$ \\
\hline d & 825 & 791 & 845 & (broad) & 785 & Bending $\mathrm{PO}_{2}$ \\
\hline
\end{tabular}


a)

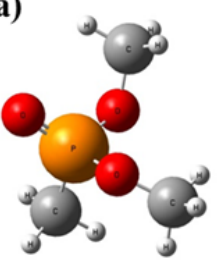

i) DMMP

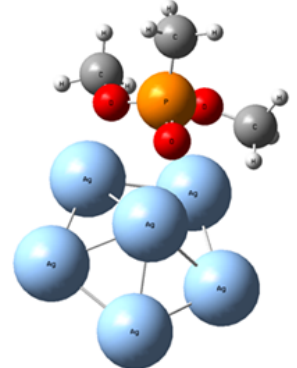

ii) $\mathrm{Ag}-\mathrm{DMMP} *\left(\mathrm{PO}_{3}\right)$

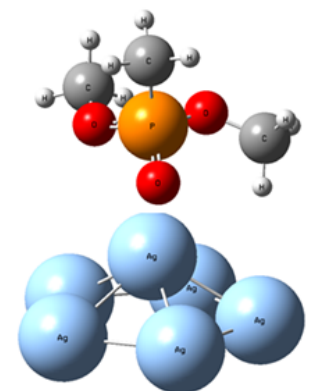

iii) $\mathrm{Ag}-\mathrm{DMMP}(\mathrm{P}=\mathrm{O})$

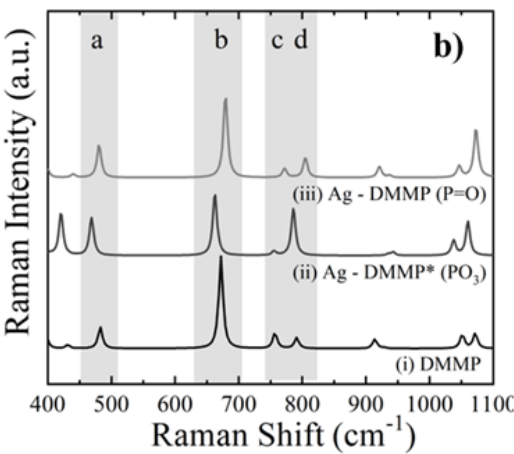

354

355

356

357

358

359

360

361

362

363

364

365

366

367

368

369

370

371

372

373

374

375

376

377

378

379

Fig. 6. a) Optimized geometries for the DMMP in vacuum (i) and absorbed on six Ag atoms according to the following possibilities: (ii) anchoring through the three oxygen atoms of DMMP $\left(\mathrm{PO}_{3}\right)$ and (iii) interaction via $\mathrm{P}=\mathrm{O}$ group. b) Calculated spectra of DMMP (i) and DMMP-Ag complexes considering de adsorption of DMMP on three silver atoms through: (ii) the three oxygens $\left(\mathrm{PO}_{3}\right)$ and (iii) $\mathrm{P}=\mathrm{O}$ group. Grey shadows indicate bands assigned to DMMP (more information in Table 1).

To evaluate the reproducibility of the Ag nplates@graphite fabrication method, three different Ag nplates@graphite substrates were prepared, and the SERS spectra (2.5ppmV DMMP) were collected randomly in ten different spots. Figure 7a shows the average peak intensity at $712 \mathrm{~cm}^{-1}$ of DMMP, showing an average relative standard deviation (RSD) for the SERS intensity of 5\%. This value indicates the good reproducibility of fabrication method and also the detection capabilities of $\mathrm{Ag}$ nPlates@graphite substrates.

Finally, we further evaluate the concept of SERS sensor for rapid CWAs detection in gas phase. Figure $7 \mathrm{~b}$ shows the intensity evolution of DMMP, monitoring peak displayed at $712 \mathrm{~cm}^{-1}$, as a function of exposure time to the vapours of this gas. It is important to note that the response time $t_{50 \%}$ defined as the time to reach $50 \%$ or $90 \%$ of the final Raman intensity, is $t_{50 \%}=137 \mathrm{~s}$ and $t_{90 \%}$ is only $261 \mathrm{~s}$. This value is outstanding compared to the early work for detection of DMMP in gas phase that needed 40 minutes exposure to acquire a meaningful spectrum with the benchtop equipment available at that time. This time is slightly higher than the response time that we found in our previous study with benchtop equipment [9]. It is also important to note that due to the lower resolution of the handheld equipment, the acquisition time is higher (100s) compared to $(1 \mathrm{~s})$ in benchtop equipment. Although it is difficult to compare detection values for different conditions, molecules and equipment's, the relevance of the obtained results it is presented in Table 2 with the available literature data for gas phase 
detection at concentrations below $10 \mathrm{ppm}$. These results demonstrate the high quality of

381 Ag nplates@graphite as SERS sensors of gas molecules, combined with handheld equipment for real applications on field.
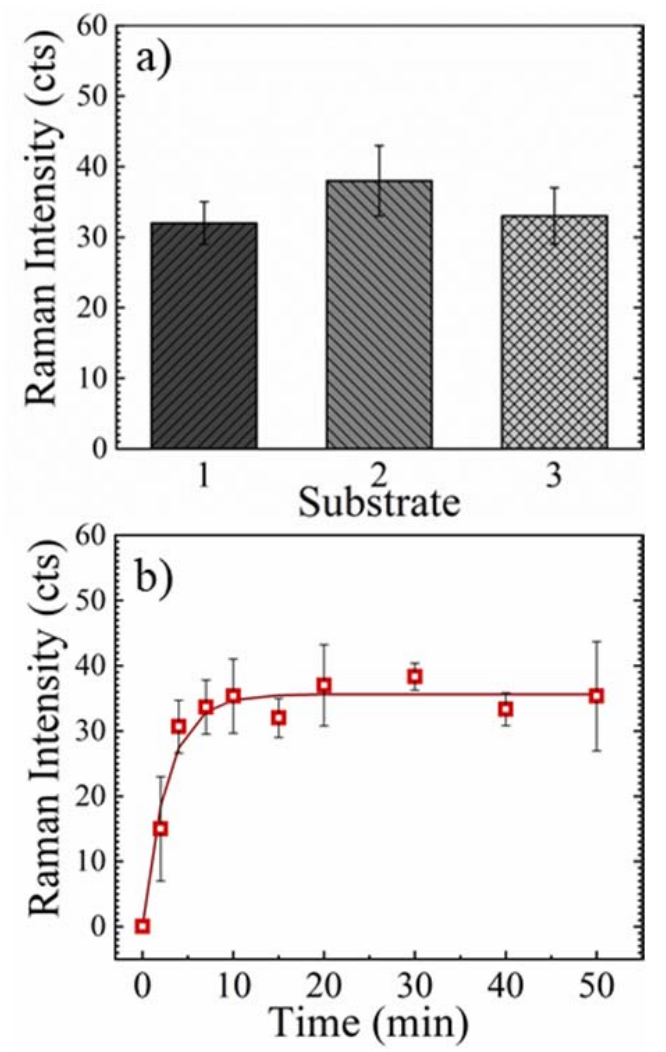

Fig. 7. a) The reproducibility of the Ag nplates@graphite: average intensity of the peak at 712 $\mathrm{cm}^{-1}$ of DMMP (2.5 ppmV) of three different Ag nplates@graphite. The error bars indicate standard deviation. b) Intensity value of the peak at $712 \mathrm{~cm}^{-1}$ of DMMP as a function of exposure time to $2.5 \mathrm{ppmV}$. Both experiments were carried out with the portable Raman equipment. 
Table 2. Literature review for gas phase detection of different molecules, at concentrations

below 10ppm, with SERS as detection technology.

\begin{tabular}{|c|c|c|c|c|c|}
\hline Material & Target molecule & $\begin{array}{l}\text { Raman } \\
\text { equipment }\end{array}$ & $\begin{array}{l}\text { Limit of } \\
\text { detection }\end{array}$ & $\begin{array}{l}\text { Detection } \\
\text { time }\end{array}$ & Ref. \\
\hline AgnPlates@graphite & DMMP & Portable & $2.5 \mathrm{ppmV}$ & $137 \mathrm{~s}$ & $\begin{array}{l}\text { This } \\
\text { work }\end{array}$ \\
\hline Cool Ag nanorods & Benzene & Portable & $10 \mathrm{ppmV}$ & $2 \mathrm{~min}$ & {$[7]$} \\
\hline $\begin{array}{l}\text { Ag@silica } \\
\text { nanospheres }\end{array}$ & $\begin{array}{l}\text { Benzene thiol } \\
\text { (BT) }\end{array}$ & Portable & $8 \mathrm{ppmV}$ & $50-100 \mathrm{~s}$ & {$[2]$} \\
\hline Au-Ag nanowires & $\mathrm{NO}_{2}$ & Portable & $100 \mathrm{ppbV}$ & $3 \mathrm{~min}$ & {$[8]$} \\
\hline Agnanorods@ $\mathrm{HfO}_{2}$ & 2-naphtalenethiol & Portable & $20 \mathrm{ppbV}$ & $40 \mathrm{~min}$ & {$[3]$} \\
\hline Gold nanopillars & $\begin{array}{l}\text { Hydrogen } \\
\text { cyanine }\end{array}$ & Benchtop & $5 \mathrm{ppmV}$ & $15 \mathrm{~min}$ & {$[5]$} \\
\hline AuNP@citrate & DMMP & Benchtop & $625 \mathrm{ppbV}$ & $120 \mathrm{~s}$ & [9] \\
\hline Agnanocubes@ZIF8 & 2-nitrotoluene & Benchtop & $50 \mathrm{ppbV}$ & $15 \mathrm{~min}$ & {$[6]$} \\
\hline
\end{tabular}

\section{Conclusions}

The effect of different substrates $\left(\mathrm{SiO}_{2} / \mathrm{Si}\right.$, stainless steel mesh and graphite foil), where plasmonic silver nanoplates were deposited, on the SERS signal, with three lasers ( $\lambda=532,633$ and $785 \mathrm{~nm}$ ) has been evaluated with two molecules R6G in liquid and DMMP, 2.5ppmV in gas phase. It has been demonstrated that graphite is the best choice because of efficient quenching of the fluorescent signal ( $\lambda=532$ and $633 \mathrm{~nm})$, via charge transfer or energy transfer. This energy transfer is also important when working with the $785 \mathrm{~nm}$ laser line, with its wavelength matching the plasmon resonance of the silver nanoplates. In this case a clear spectrum of R6G could be only distinguished on graphite support. When working with DMMP, it was only possible to use the $785 \mathrm{~nm}$ laser in combination with graphite, otherwise the high energy concentrated on the sample during measurement decomposed the adsorbed DMMP molecules. The interactions of the DMMP molecule with the silver surface modify the intensities and positions of the

412 vibrational Raman bands. DFT simulations suggest such variations by the formation of

413 Ag-DMMP complexes via the $\mathrm{PO}_{3}$ umbrella. The SERS measurements carried out in different prepared samples with the handheld Raman equipment for $2.5 \mathrm{ppmV}$ in gas phase showed a fast response and reproducible fingerprint of the molecule and relative 
416 standard deviation of SERS intensity signal of 5\%, paving the way for on field 417 applications.

\section{Conflict of interest:}

419 Authors declare that there are no conflicts of interest.

\section{Acknowledgements}

421 Authors are grateful for financial support from the European Union's Horizon 2020 422 research and innovation programme under the Marie Sklodowska-Curie grant agreement Nr. 823895, MICINN (CTQ2013-49068-C2-1-R and CTQ2016-79419-R), 424 Gobierno de Aragón (T57-17R p), Feder 2014-2020 "Construyendo Europa desde Aragón") and CIBER-BNN (initiative funded by the VI National R\&D\&i Plan 20082011, Iniciativa Ingenio 2010, Consolider Program, CIBER Actions and financed by the Instituto de Salud Carlos III with assistance from the European Regional Development Fund). Authors would like to acknowledge the use of Servicio General de Apoyo a la Investigación-SAI, Universidad de Zaragoza. The microcopy works have been conducted in the Laboratorio de Microscopias Avanzadas at Instituto de Nanociencia de Aragon-Universidad de Zaragoza. Authors acknowledge the LMA-INA for offering access to their instruments and expertise.

\section{References}

[1] A. Hakonen, P.O. Andersson, M. Stenbæk Schmidt, T. Rindzevicius, M. Käll,

[2] K.B. Biggs, J.P. Camden, J.N. Anker, R.P.V. Duyne, Surface-enhanced raman spectroscopy of benzenethiol adsorbed from the gas phase onto silver film over nanosphere surfaces: Determination of the sticking probability and detection limit time, J. Phys. Chem. A. 113 (2009) 4581-4586. doi:10.1021/jp8112649.

[3] L. Ma, H. Wu, Y. Huang, S. Zou, J. Li, Z. Zhang, High-Performance Real-Time SERS Detection with Recyclable Ag Nanorods@HfO2 Substrates, ACS Appl. Mater. Interfaces. 8 (2016) 27162-27168. doi:10.1021/acsami.6b10818.

[4] R.K. Lauridsen, T. Rindzevicius, S. Molin, H.K. Johansen, R.W. Berg, T.S. 
Alstrøm, K. Almdal, F. Larsen, M.S. Schmidt, A. Boisen, Towards quantitative SERS detection of hydrogen cyanide at ppb level for human breath analysis, Sens. Bio-Sensing Res. 5 (2015) 84-89. doi:10.1016/j.sbsr.2015.07.002.

[5] R.K. Lauridsen, P.B. Skou, T. Rindzevicius, K. Wu, S. Molin, S.B. Engelsen, K.G. Nielsen, H.K. Johansen, A. Boisen, SERS spectroscopy for detection of hydrogen cyanide in breath from children colonised with: P. aeruginosa, Anal. Methods. 9 (2017) 5757-5762. doi:10.1039/c7ay01693j.

[6] C.S.L. Koh, H.K. Lee, X. Han, H.Y.F. Sim, X.Y. Ling, Plasmonic nose: Integrating the MOF-enabled molecular preconcentration effect with a plasmonic array for recognition of molecular-level volatile organic compounds, Chem. Commun. 54 (2018) 2546-2549. doi:10.1039/c8cc00564h.

[7] M.K. Oh, R. De, S.Y. Yim, Highly sensitive VOC gas sensor employing deep cooling of SERS film, J. Raman Spectrosc. 49 (2018) 800-809. doi: $10.1002 /$ jrs.5355.

[8] S. Kim, D.H. Kim, S.G. Park, Highly sensitive and on-site NO 2 SERS sensors operated under ambient conditions, Analyst. 143 (2018) 3006-3010. doi:10.1039/c8an00845k.

[9] M. Lafuente, I. Pellejero, V. Sebastián, M.A. Urbiztondo, R. Mallada, M.P. Pina, J. Santamaría, Highly sensitive SERS quantification of organophosphorous chemical warfare agents: A major step towards the real time sensing in the gas phase, Sensors Actuators, B Chem. 267 (2018). doi:10.1016/j.snb.2018.04.058.

[10] E. Le Ru, P.G. Etchegoin, Principles of Surface-Enhanced Raman Spectroscopy: and related plasmonic effects., Elsevier, 2009.

[11] N. Taranenko, J.-P. Alarie, D.L. Stokes, T. Vo-Dinh, Surface-Enhanced Raman Detection of Nerve Agent Simulant (DMMP and DIMP) Vapor on Electrochemically Prepared Silver Oxide Substrates, J. Raman Spectrosc. 27 (1996) 379-384. doi:10.1002/(SICI)1097-4555(199605)27:5<379::AIDJRS925>3.0.CO;2-G.

[12] M.J. Frisch, G.W. Trucks, H.B. Schlegel, G.E. Scuseria, M.A. Robb, J.R. Cheeseman, G. Scalmani, V. Barone, B. Mennucci, G.A. Petersson, Gaussian 09, 
[13] A.J. Frank, N. Cathcart, K.E. Maly, V. Kitaev, Synthesis of silver nanoprisms with variable size and investigation of their optical properties: A first-year undergraduate experiment exploring plasmonic nanoparticles, J. Chem. Educ. 87 (2010) 1098-1101. doi:10.1021/ed100166g.

[14] E.C. Le Ru, E. Blackie, M. Meyer, P.G. Etchegoint, Surface enhanced raman scattering enhancement factors: A comprehensive study, J. Phys. Chem. C. 111 (2007) 13794-13803. doi:10.1021/jp0687908.

[15] A.D. Becke, Density-functional thermochemistry. III. The role of exact exchange, J. Chem. Phys. 98 (1993) 5648-5652. doi:10.1063/1.464913.

[16] P.J. Stephens, F.J. Devlin, C.F. Chabalowski, M.J. Frisch, Ab Initio calculation of vibrational absorption and circular dichroism spectra using density functional force fields, J. Phys. Chem. 98 (1994) 11623-11627. doi:10.1021/j100096a001.

[17] H. Hou, P. Wang, J. Zhang, C. Li, Y. Jin, Graphene Oxide-Supported Ag Nanoplates as LSPR Tunable and Reproducible Substrates for SERS Applications with Optimized Sensitivity, ACS Appl. Mater. Interfaces. 7 (2015) 18038-18045. doi:10.1021/acsami.5b04946.

[18] X. Jiang, Q. Zeng, A. Yu, Thiol-frozen shape evolution of triangular silver nanoplates, Langmuir. 23 (2007) 2218-2223. doi:10.1021/la062797z.

[19] J. Lee, B. Hua, S. Park, M. Ha, Y. Lee, Z. Fan, H. Ko, Tailoring surface plasmons of high-density gold nanostar assemblies on metal films for surfaceenhanced Raman spectroscopy, Nanoscale. 6 (2014) 616-623. doi:10.1039/c3nr04752k.

[20] S.K. Ghosh, S. Nath, S. Kundu, K. Esumi, T. Pal, Solvent and ligand effects on the localized surface plasmon resonance (LSPR) of gold colloids, J. Phys. Chem. B. 108 (2004) 13963-13971. doi:10.1021/jp047021q.

[21] F.J. Beck, E. Verhagen, S. Mokkapati, A. Polman, K.R. Catchpole, Resonant SPP modes supported by discrete metal nanoparticles on high-index substrates, Opt. Express. 19 (2011) A146. doi:10.1364/oe.19.00a146. 
[22] M.W. Knight, Y. Wu, J.B. Lassiter, P. Nordlander, N.J. Halas, Substrates matter: influence of an adjacent dielectric on an individual plasmonic nanoparticle, Nano Lett. 9 (2009) 2188-2192. doi:10.1021/n1900945q.

[23] S. Sil, N. Kuhar, S. Acharya, S. Umapathy, Is chemically synthesized graphene "Really" a unique substrate for SERS and Fluorescence quenching?, Sci. Rep. 3 (2013) 1-6. doi:10.1038/srep03336.

[24] M. Lafuente, E.J.W. Berenschot, R.M. Tiggelaar, R. Mallada, N.R. Tas, M.P. Pina, 3D fractals as SERS active platforms: Preparation and evaluation for gas phase detection of G-nerve agents, Micromachines. 9 (2018). doi:10.3390/mi9020060.

[25] J.C.S. Costa, R.A. Ando, A.C. Sant'Ana, P. Corio, Surface-enhanced Raman spectroscopy studies of organophosphorous model molecules and pesticides, Phys. Chem. Chem. Phys. 14 (2012) 15645-15651. doi:10.1039/c2cp42496g. 
Marta Lafuente ${ }^{\text {a,b }}$, Diego Sanz ${ }^{\text {a,b }}$, Miguel Urbiztondo ${ }^{c}$, Jesús Santamaria a,b,d , María Pilar Pina ${ }^{\text {a,b,d }}$, Reyes Mallada ${ }^{a, b, d, *}$

${ }^{a}$ Nanoscience Institute of Aragon (INA), University of Zaragoza, Department of Chemical \& Environmental Engineering, Edificio I+D+i, Campus Rio Ebro, C/Mariano Esquillor s/n, 50018 Zaragoza, Spain

${ }^{b}$ Instituto de Ciencia de Materiales de Aragón (ICMA), Universidad de Zaragoza-CSIC, 50009 Zaragoza, Spain.

${ }^{c}$ Centro Universitario de la Defensa de Zaragoza, Carretera Huesca s/n, 50090 Zaragoza, Spain.

${ }^{d}$ Networking Research Center on Bioengineering, Biomaterials and Nanomedicine, CIBERBBN, 28029 Madrid, Spain.

537

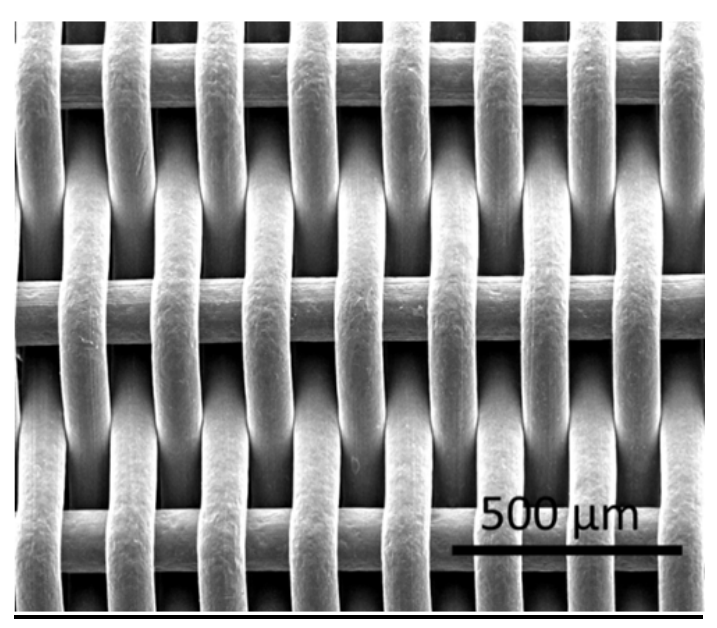

Figure S1. SEM image of the stainless steel mesh used as SERS substrate support. 
a)

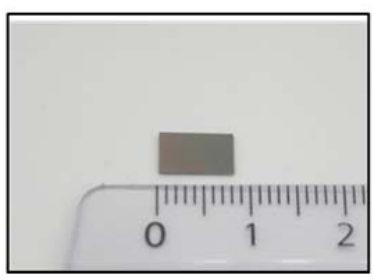

b)

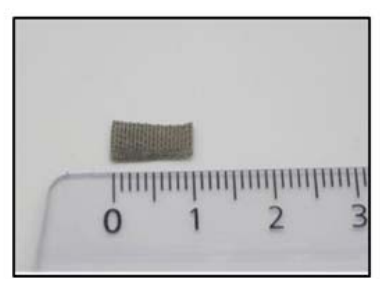

c)

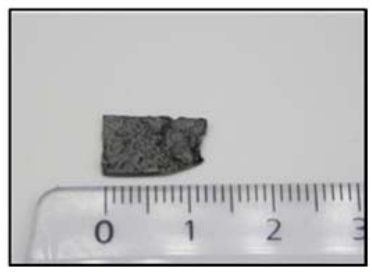

549

Figure S2. Digital photographs of the three different supports used as SERS substrate:

550

a) $\mathrm{SiO}_{2} / \mathrm{Si}$ chip; b) stainless steel mesh and c) graphite foil. Rule in centimeters $(\mathrm{cm})$.

552

553 
Figure S3.

556
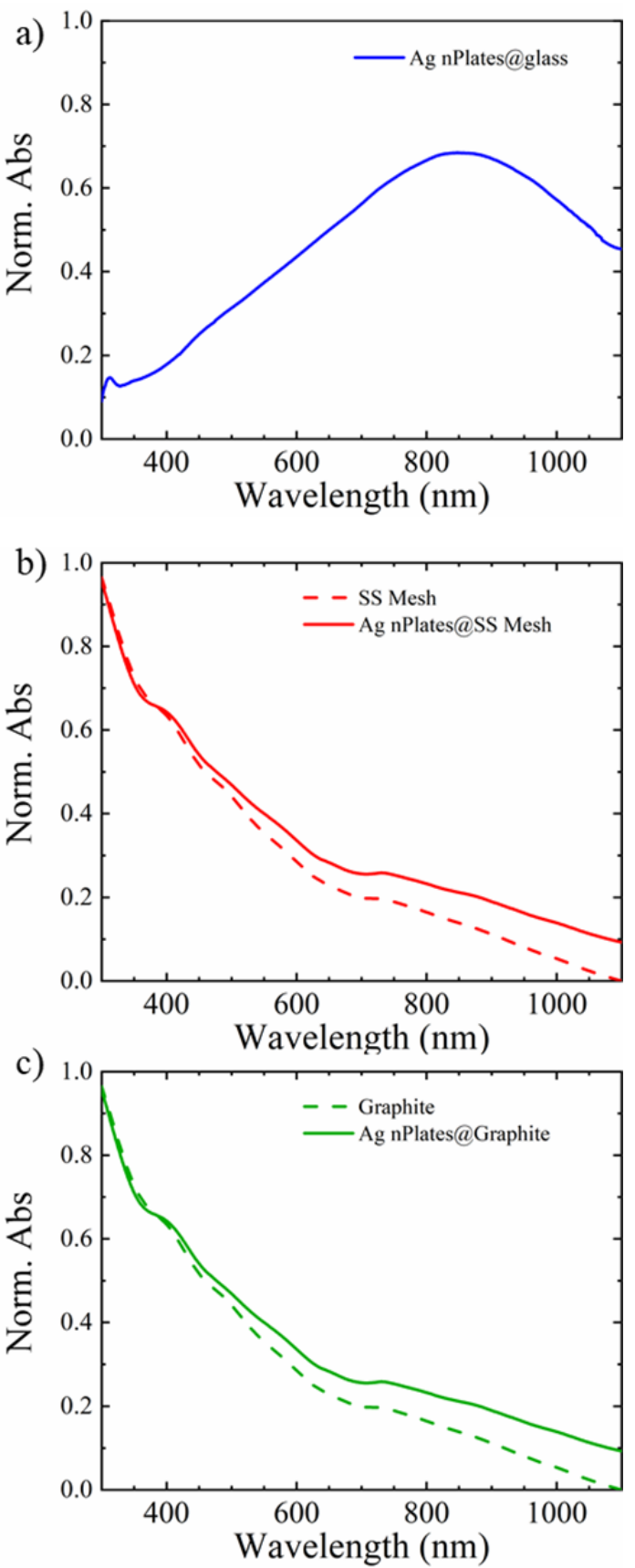

557

558 Figure S3. UV-Vis spectra of deposited Ag nanoplates on (a) glass; (b) Ag nPlates@SS

559 Mesh and (c) graphite. The spectra of mesh and graphite before incorporating Ag 560 nPlates are also plotted. 

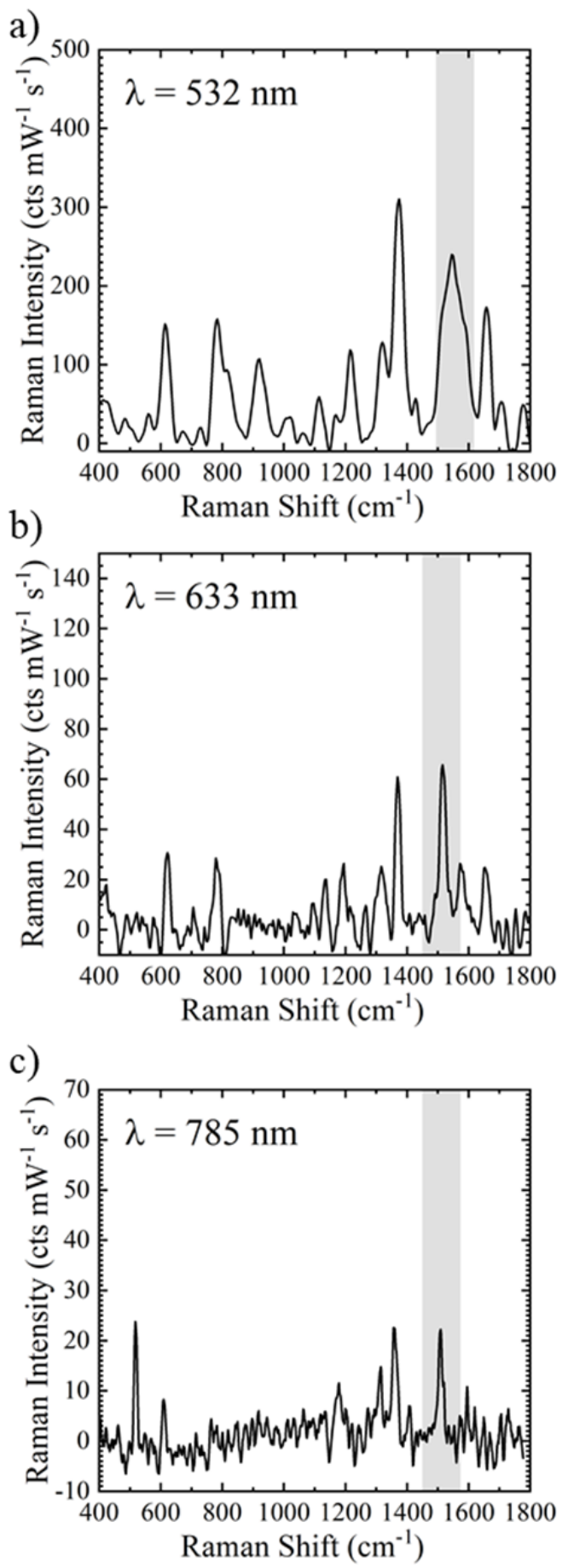

563

564 Figure S4. Raman spectra of Rhodamine 6G (R6G, 1mM) measured under the three different 565 excitation wavelengths: a) $532 \mathrm{~nm}$; b) $633 \mathrm{~nm}$ and c) $785 \mathrm{~nm}$. Grey shadow indicates vibrational 566 mode selected for AEF calculations: PC stretching around $1512 \mathrm{~cm}^{-1}$. 